\title{
Solar oscillations
}

\author{
H. M. Antia \\ Tata Institute of Fundamental Research, Homi Bhabha Road, Mumbai 400005, India \\ email: antia@tifr.res.in
}

\begin{abstract}
Study of solar oscillations has provided us detailed information about solar structure and dynamics. These in turn provide a test of theories of stellar structure and evolution as well as theories of angular momentum transfer and dynamo. Some of these results about the solar structure and its implication on the recent revision of heavy element abundances are described. Apart from these the solar cycle variations in the rotation rate and its gradients are also discussed.
\end{abstract}

Keywords. Sun: abundances, Sun: helioseismology, Sun: interior, Sun: oscillations, Sun: rotation

\section{Introduction}

The Sun oscillates in a set of well defined discrete frequencies, which can be observed at the solar surface. These frequencies are determined by the internal structure and dynamics and hence they contain information about the internal structure and dynamics. During the last solar cycle, detailed observations of solar oscillations have provided a unique opportunity to study the solar structure and dynamics (see Christensen-Dalsgaard 2002 for a review). A detailed seismic study of solar structure led to significant improvements in theoretical solar models and with recent input physics the solar model agreed well with the seismically inferred solar structure (Christensen-Dalsgaard et al. 1996; Gough et al. 1996). The improvements in solar models include the improvements in input physics like, the equation of state, opacities and nuclear reaction rates as well as the inclusion of diffusion of helium and heavy elements in the solar interior (Christensen-Dalsgaard et al. 1993). As a result of these developments it became clear that the discrepancy between the observed flux of solar neutrinos and those calculated in a solar model should be due to neutrino oscillations. In fact, the discrepancy in solar neutrino fluxes was another motivation for extensive tests of solar models, which also contributed to some of these improvements. The neutrino oscillations are now confirmed by measurements from the Sudbury Neutrino Observatory (Ahmad et al. 2002). However, this excellent agreement between the standard solar model and seismically inferred structure was spoilt when Asplund et al. (2004) found that the Oxygen abundance in the solar photosphere should be reduced by a factor of 1.5. This has led to a crisis in solar models, which would be discussed in Section 2.

Apart from solar structure the frequencies of solar oscillations also give us information about the rotation rate in the solar interior (e.g., Thompson et al. 1996; Schou et al. 1998). These results established that the differential rotation observed at the solar surface continues through the solar convection zone, while most of the radiative interior has nearly constant rotation rate. There is a sharp transition between these two regions near the base of the convection zone and this transition region has been named tachocline (Spiegel \& Zahn 1992). Because of the strong shear in the tachocline region, it is the favoured location for the operation of the solar dynamo. Currently, the major sources of seismic data are (1) the Global Oscillation Network Group (GONG) which is 
a network of six sites around the world (Hill et al. 1996) that is operating since May 1995 and (2) the Michelson Doppler Imager (MDI) instrument (Scherrer et al. 1995) on board the SOHO satellite which is operating since May 1996. With the availability of seismic data for the last 13 years it has become possible to study the temporal variations over the solar cycle. In particular, the inferred temporal variations in the solar rotation rate can provide a crucial test of dynamo models.

\section{Solar structure and photospheric abundances}

Fig. 1 shows the relative differences in sound speed and density between a standard solar model of Christensen-Dalsgaard et al. (1996) and the Sun as inferred from seismic data. This solar model was constructed using diffusion of helium and heavy elements below the convection zone and used the heavy element abundances from Grevesse \& Noels (1993). It can be seen that there is very good agreement and the difference in sound speed is generally less than $0.1 \%$. The two major regions of discrepancy are near the surface and near the base of the convection zone. There is some difference in the core also, but in that region the errors in inversions are somewhat large and it is not clear if the difference is indeed significant, particularly when systematic errors in inversion are considered. The sharp peak near the base of the convection zone $\left(r=0.713 R_{\odot}\right)$ has been attributed to mixing in the tachocline region, and solar models (e.g., Brun et al. 1999) which include some mixing in this region do not show this peak. The dip near the surface is most likely to be due to improper estimate of solar radius. If the radial distance, $r$ in the model is scaled by a factor of 1.00018 (i.e., $r / 1.00018$ ) before taking differences then this dip is substantially reduced as seen by the dashed curve in Fig. 1. However, a solar model with a different radius also shows similar dip and hence it is not due to use of incorrect solar radius, but rather because of the uncertainties in treatment of outer layers, the position of solar surface is not correctly estimated in a solar model. The generally adopted value of the solar radius $(695.99 \mathrm{Mm})$ from observations should refer to a layer in the atmosphere about $500 \mathrm{~km}$ above the layer with unit optical depth which is normally used as definition of solar surface in a solar model (Brown \& ChristensenDalsgaard 1998). Thus a different value of solar radius should be used in a solar model. This value can be calibrated using frequencies of f-modes (Schou et al. 1997; Antia 1998) and gives a value 200 to $300 \mathrm{~km}$ less than the standard value. This is about $200 \mathrm{~km}$ larger than the value that is expected and this discrepancy is the one which gives the dip in Fig. 1. Of course, the use of the revised value of radius in a solar model will not change Fig. 1 substantially as the dip is due to inadequacy in solar models near the surface and cannot be eliminated by changing the solar radius.

Fig. 1 shows the differences with respect to a solar model with old chemical composition, which has been revised since then. Abundances of most heavy elements are determined spectroscopically and requires a model of solar atmosphere. Traditionally, these solar atmospheric models are 1 dimensional as they assume spherical symmetry. The effect of turbulence is incorporated through ad hoc parameters like micro and macroturbulence. With increase in computing power it has become possible to make limited 3D models of solar atmosphere which attempt to include turbulence over a limited range of length scales in the calculations. Using such models Asplund et al. (2004) calculated the abundances of oxygen and found that it needs to be reduced by a factor of almost 1.5. Similarly, abundances of many other elements were also reduced by similar factors. As a result of these reduction the value of $Z / X$ in the Sun reduced from 0.023 (Grevesse \& Sauval 1998, henceforth GS98) to 0.0165 (Asplund et al. 2005, henceforth AGS05). As a result the opacity in the solar interior is substantially reduced and the structure of 

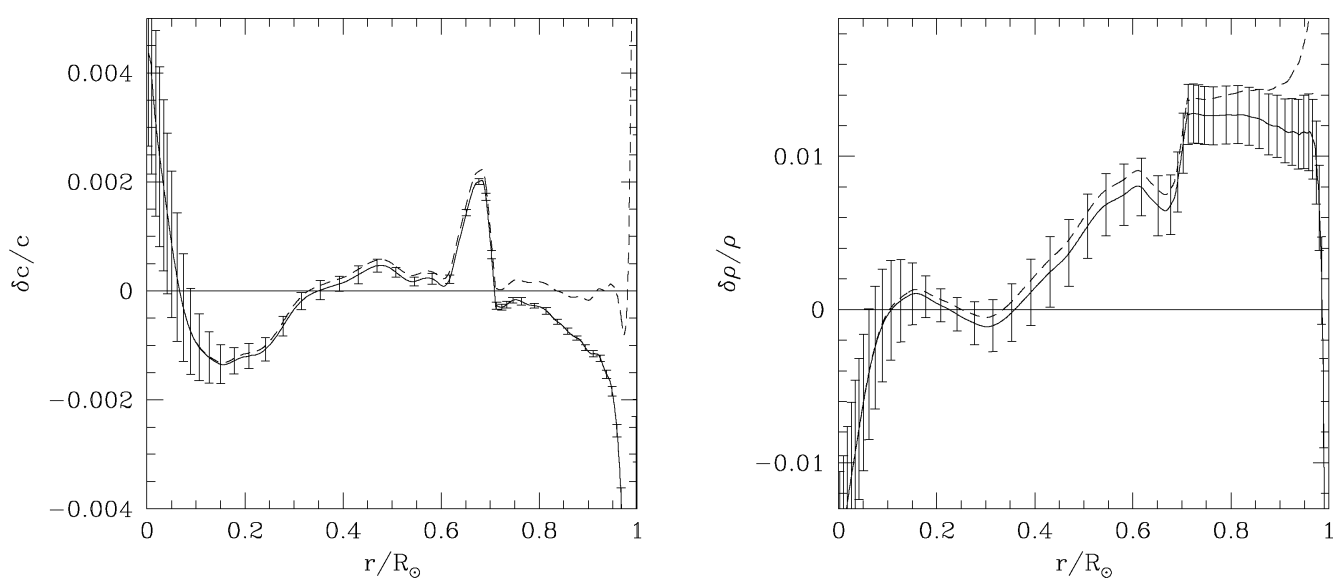

Figure 1. Relative differences in sound speed and density between the standard solar model of Christensen-Dalsgaard et al. (1996) and the Sun as inferred from seismic data from GONG. The dashed line shows the difference after scaling the model radius by a factor of 1.00018 before taking the difference.

resulting solar models is quite different from the seismically inferred structure (Bahcall \& Pinsonneault 2004; Basu \& Antia 2004; Turck-Chiéze et al. 2004). Apart from the increased difference in structure variables like the sound speed and density, the depth of the convection zone as well as the helium abundance in the convection zone, reduces substantially below the seismically measured values. Bahcall et al. (2006) have done a detailed Monte-Carlo simulations by constructing solar models where various input parameters (including abundances) are randomly varied within the estimated errors to find that solar models with mean heavy element abundances from GS98 are consistent with seismic constraints on the depth of the convection zone and its helium abundance. While models with abundances from AGS05 are not consistent with these constraints. In fact, long before the current revision in $Z$, low $Z$ solar models were postulated to lower the solar neutrino flux, but such models were ruled out from seismic constraints (e.g., Christensen-Dalsgaard $\tilde{\&}$ Gough 1980). Similarly, from a detailed study of the depth of the convection zone, Basu \& Antia (1997) concluded that $Z=0.0245 \pm 0.0008$ assuming that the OPAL opacities were valid. This study did not include the effect of varying mixture of heavy elements, but that effect is found to be small (Basu \& Antia 2004).

Numerous attempts have been made to modify the solar models to restore the good agreement between solar models and seismic data. These include increase in opacities (Basu \& Antia 2004; Bahcall et al. 2004, 2005a), increasing the rate of diffusion of heavy elements below the base of the convection zone (e.g., Basu \& Antia 2004; Guzik et al. 2005), accretion of low $Z$ material during solar evolution (Castro et al. 2007), increasing the abundance of Ne (Antia \& Basu 2005; Bahcall et al. 2005b). However, none of these attempts have been successful, in the sense that required variations are beyond reasonable estimates and even if a combination of these effects is considered, the resulting solar model doesn't match the seismic structure in full details. For example, the required opacity increase is by $11-25 \%$ over the OPAL values (Rogers \& Iglesias 1992; Iglesias \& Rogers 1996), while recent independent computation of opacities by OP project (Badnell et al. 2005) gives a difference of less than $2 \%$ near the base of the convection zone. Thus it is unlikely that opacities can be increased by the required amount.

Since the main cause of discrepancy in solar models is the reduction in opacities, Antia \& Basu (2005) examined the effect of abundances of different elements on opacities 


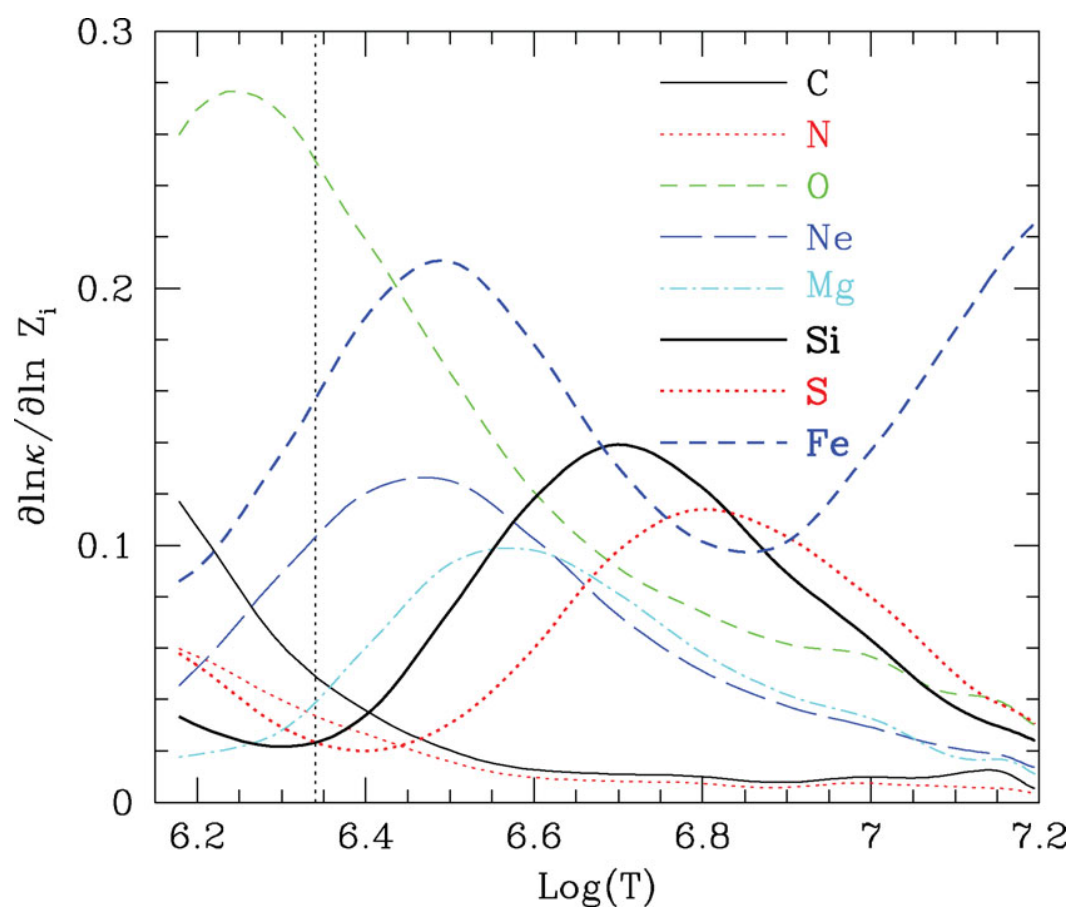

Figure 2. Logarithmic derivative of opacity with respect to abundances of individual heavy elements in a solar model. The OPAL opacity tables are used to calculate the derivatives. The dashed vertical line marks the position of the base of the convection zone.

in the radiative interior. Fig. 2 shows the logarithmic derivatives of opacity with respect to abundances of some of the dominant heavy elements. Apart from oxygen, which plays the most dominant role in opacity near the base of the convection zone, Iron and Neon are also important contributors. Other elements were not found to make significant contributions to opacity in the required region. Of these the Neon abundance in the photosphere cannot be determined directly as Neon doesn't form any line in the photosphere. Thus Neon abundance is determined from coronal lines or solar wind which generally determines the ratio of $\mathrm{Ne} / \mathrm{O}$ abundances. Hence a reduction in $\mathrm{O}$ abundance automatically reduced the $\mathrm{Ne}$ abundance. It is well-known that the abundances in the corona/ solar wind are not the same as photospheric abundances as there is the wellknown effect of First Ionisation Potential (FIP), the mechanism for which is not understood. Elements with high FIP are known to be depleted in corona or solar wind. Since $\mathrm{O}$ and Ne both have relatively high FIP their relative abundance is not expected to be affected by this effect, but that is merely an assumption as the FIP of Ne is almost a factor of 2 higher than that of $\mathrm{O}$ and there are no other elements with similar FIP as Ne, whose abundances are independently known for calibrating the FIP effect. Further, it is well known that abundance of helium, which also has high FIP and doesn't form lines in photosphere, was underestimated through similar procedure (Anders \& Grevesse 1989). The helium abundance was ultimately determined using seismic data. While the coronal or solar wind measurements give the abundance ratios, for solar models we need absolute value of abundances and it may not be possible to find abundances that are consistent with all abundance ratios. For example, the Ne abundance as determined from $\mathrm{Ne} / \mathrm{O}$ ratio doesn't agree with that from $\mathrm{Ne} / \mathrm{Mg}$ ratio after compensating 
for FIP effect (Feldman \& Widing 2003). Considering all these uncertainties, it was suggested that $\mathrm{Ne}$ abundance may be increased to compensate for a reduction in oxygen abundance.

It can be easily estimated that in order to compensate for a reduction in oxygen abundance by a factor of 1.5 the neon abundance needs to be increased by a factor of 4 to restore the opacity near the base of the convection zone. Such an increase in neon abundance is clearly unacceptable, and even after that the structure of solar model in the core will be significantly different (Bahcall et al. 2005b). However, if the CNO abundances are increased by $1 \sigma$ of their respective values determined by AGS05, then the required increase in neon abundance is about a factor of 2.5, which is of the same order as the difference between the GS98 and AGS05 values. Soon after this suggestion was made Drake \& Testa (2005) measured the Ne/O abundance in nearby stars using Chandra observations to find a value that is a factor of 2.7 higher than that used by AGS05. However, a reanalysis of solar data by Schmelz et al. (2005) and Young (2005) found results consistent with AGS05 value and they attributed the higher value found by Drake \& Testa to be due to choice of stars with higher activity level. Recently, through a survey of $\mathrm{Ne} / \mathrm{O}$ coronal abundances in a number of late type stars, Garcia-Alvarez et al. (2008) have claimed that $\mathrm{Ne} / \mathrm{O}$ abundance determined from coronal lines approaches the photospheric value at higher activity levels, thus supporting the higher value of $\mathrm{Ne} / \mathrm{O}$ determined by Drake \& Testa. Many other measurements of Ne abundance in the Sun and other related astrophysical objects have given conflicting results and the issue is not resolved (cf., Basu \& Antia 2008).

Since there is considerable uncertainty in spectroscopic determination of heavy element abundances, it is interesting to investigate if it is possible to determine these abundances using seismic techniques similar to those used for determining helium abundance. The helium abundance estimates are obtained from sound speed in the HeII ionisation zone, where the adiabatic index $\Gamma_{1}$ is reduced below its normal value of $5 / 3$ and the extent of reduction depends on the $\mathrm{He}$ abundance. It is often convenient to use the dimensionless gradient of sound speed $W(r)=(1 / g) d c^{2} / d r$, where $g$ is the acceleration due to gravity and $c$ is the sound speed. The function $W(r)$ shows a peak in the HeII ionisation zone which can be calibrated to determine helium abundance (Gough 1984; Basu \& Antia 1995). In principle, the same technique can be applied to determination of heavy element abundances, but the main difficulties are that first these abundances (by number) are two orders of magnitude smaller and hence the effect is very small and second the ionisation zones of various elements overlap and it is difficult to isolate the effect of each element. Nevertheless, after detailed study, Antia \& Basu (2006) found that it should be possible to determine the total heavy element abundance, $Z$ using this technique and found a value of $Z=0.0172 \pm 0.002$, which is consistent with GS98 value, but higher than the AGS05 value. This value is also sensitive to the equation of state, but the errorbars in the above estimate also include this effect. This effect is estimated by using various modern equations of state. This provides an independent seismic estimate for $Z$ as other estimates (e.g., Delahaye \& Pinsonneault 2006; Chaplin et al. 2007) are mainly based on effect of opacity on solar models. Thus all seismic estimates point to higher value of $Z$ that is not consistent with AGS05. The function $W(r)$ can also be used to check if increasing Ne abundance can help to compensate for reduction in $\mathrm{O}$ abundance. It may be recalled that this suggestion was based on opacities. It turns out that solar models with AGS05 oxygen abundance are not consistent with observed $W(r)$ even after increasing Ne abundance (Fig. 3). Thus increasing the neon abundance is not likely to solve the problem with solar models in all respects, but if the neon abundance is increased over the GS98 abundances it may help in improving the solar models. 


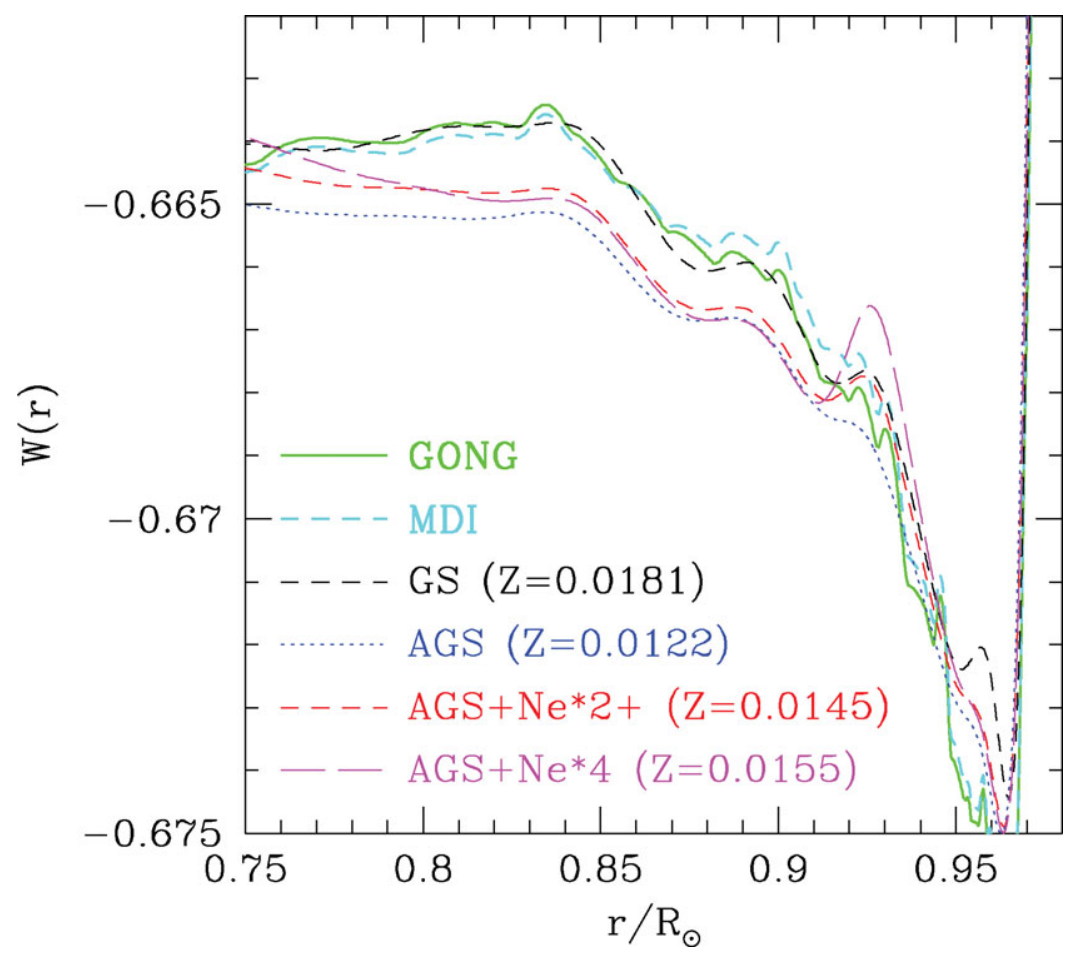

Figure 3. Logarithmic derivative of squared sound speed, $W(r)$ in a few solar models is compared with that inferred from GONG and MDI data. The $Z=0.0145$ model corresponds to the case where $\mathrm{Ne}$ abundance is increased by a factor of 2 and the $\mathrm{CNO}$ abundances are increased by $1 \sigma$ over the AGS05 abundances.

Thus seismic data consistently points to a higher oxygen abundance and if the lower abundances of AGS05 are indeed true, then it will require modifications in almost all input physics, like, opacities, equation of state, diffusion of helium and heavy elements to get the solar models in agreement with seismic data. It may be noted that some recent abundance determinations, e.g., Centeno \& Socas-Navarro (2008) using the Ni/O ratio from the blended line and Caffau et al. (2008) and Ayres (2008) using an independent 3D atmospheric model also support higher oxygen abundance close to the GS98 value. A part of difference could be due to treatment of non local thermodynamic equilibrium in atmospheric models. More work is clearly required to determine abundances reliably.

\section{Rotation in solar interior}

The rotational splitting in the frequencies of solar oscillations can be used to infer the rotation rate in the solar interior (e.g., Thompson et al. 1996; Schou et al. 1998). Such studies give the rotation rate as a function of radius and latitude over most of the solar interior. The reliability degrades as we move towards the core or high latitudes. Further, these studies only give the north-south symmetric component of the rotation rate. North-south asymmetry in the rotation rate in the outer layers can be studied using local helioseismology but we will not consider that in this review. These results have given us the well-known picture of rotation in the solar interior, where the differential rotation continues through the convection zone and near the base of the convection zone, in the 

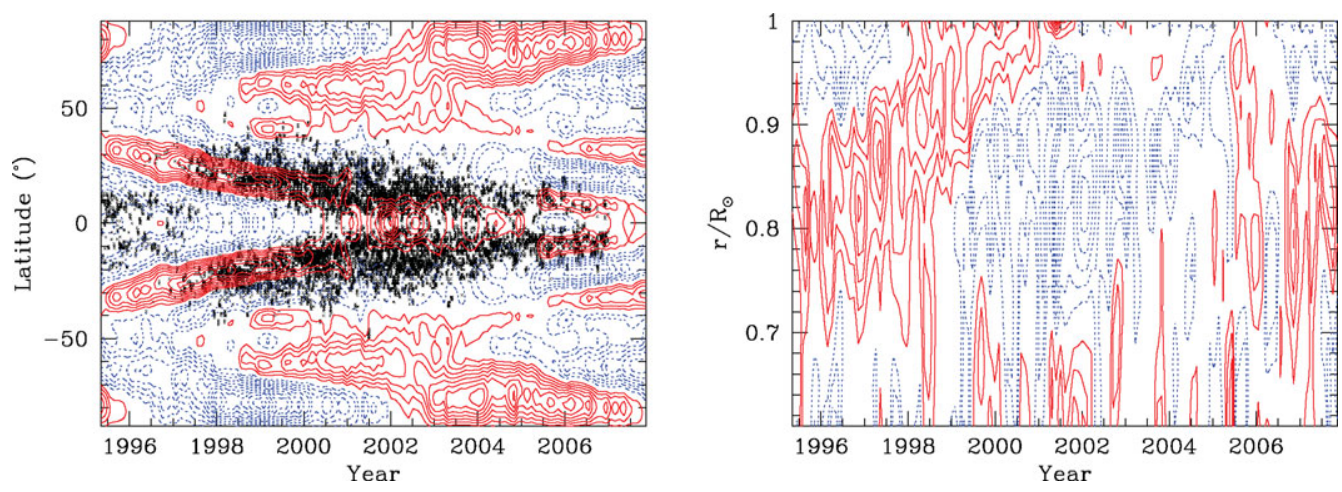

Figure 4. Contours of constant zonal flow velocity, $\delta v_{\phi}$ at $0.98 R_{\odot}$ as a function of time and latitude (Left Panel) and at a latitude of $15^{\circ}$ as a function of time and radius (Right Panel). The contour spacing is $1 \mathrm{~m} / \mathrm{s}$. Solid contours represent positive values, dotted contours show negative value. The zero contour is not shown. In the left panels the points mark the positions of sunspots.

tachocline region, there is a rather sharp transition to nearly uniform rotation in the radiative interior. Apart from this there is also a distinct shear layer near the surface where the rotation rate increases with depth. This shear layer extends to a radius of about $0.95 R_{\odot}$.

With accumulation of GONG and MDI data over the last 13 years it is also possible to study the temporal variations in the rotation rate over the solar cycle. Such studies (e.g., Howe et al. 2000; Antia \& Basu 2000, 2001; Vorontsov et al. 2002) have confirmed the existence of bands of faster and slower than average rotation in the solar interior. This pattern is similar to the torsional oscillations observed at the solar surface (Howard \& LaBonte 1986; Ulrich et al. 1988) and are referred to as zonal flow. The zonal flow velocity is obtained by subtracting the temporal mean from the rotation rate to get the residual

$$
\delta \Omega(r, \theta, t)=\Omega(r, \theta, t)-\langle\Omega(r, \theta, t)\rangle,
$$

where $\theta$ is the latitude and the angular brackets denote temporal average over the period that the data are available. To account for systematic differences in rotation rate inferred from the GONG and MDI data, the averaging is done separately for GONG and MDI data. The residual $\delta \Omega$ is essentially, the temporally varying component of $\Omega$. The bands of faster or slower than average rotation are found to move towards the equator with time at low latitudes, while at high latitudes they appear to move towards the poles. Further, this pattern penetrates to the base of the convection zone (Vorontsov et al. 2002; Basu \& Antia 2003). Fig. 4, shows cuts at $r=0.98 R_{\odot}$ and $\theta=15^{\circ}$ in the zonal flow velocity $\delta v_{\phi}=\delta \Omega r \cos \theta$. It can be seen that at low latitudes, the pattern is rising upwards at a rate of about $1 \mathrm{~m} \mathrm{~s}^{-1}$. The amplitude of temporal variation in the rotation rate is of the order of a few $\mathrm{nHz}$, which is about $0.5 \%$ of the mean rotation rate. While there are significant differences between the temporally averaged rotation rate inferred from GONG and MDI data (Schou et al. 2002), these differences essentially cancel when the temporal mean is subtracted while calculating the zonal flow velocity $\delta \Omega$. Thus there is a good agreement between GONG and MDI results for $\delta \Omega$. Below the base of the convection zone, it is difficult to infer any reliable pattern of temporal variation, because of large errors in inversion results. Near the surface, the zonal flow pattern is well correlated to the butterfly diagram representing surface magnetic field (e.g., Snodgrass 1987; Sivaraman et al. 2008), as can be seen in Fig. 4, which compares the zonal flow pattern from GONG 

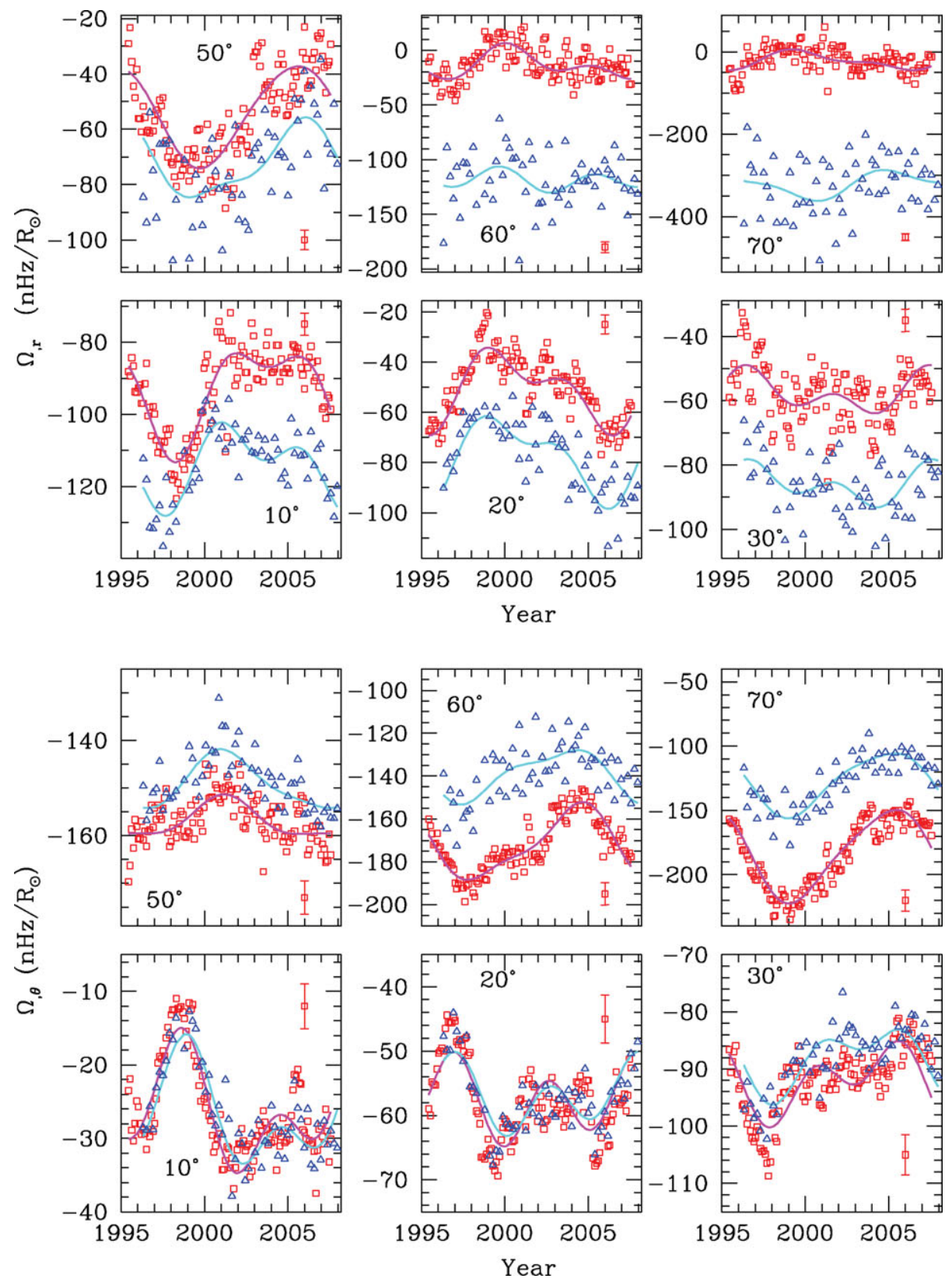

Figure 5. The radial and latitudinal gradients of the rotation rate at $r=0.95 R_{\odot}$ are shown at a few selected latitudes as a function of time for both GONG (squares) and MDI (triangles) data. For clarity the errorbars are not shown on all points but a sample errorbar is shown in the right corner in each panel.

data with the position of sunspots. The sunspots are generally concentrated in the region around the high latitude edge of the band representing faster than average rotation rate. The zonal flow pattern can also be used to test dynamo models, e.g., Covas et al. (2000) 
using a mean field dynamo model found variations in rotation rate which qualitatively resemble the observed zonal flow pattern.

The zonal flow pattern in the convection zone is well established (e.g., Howe et al. 2006; Antia et al. 2008), but for the solar dynamo the gradients of rotation rate are more relevant and hence we need to study these also. The inferred rotation rate can be differentiated to find the gradients, though the errors will be magnified during the process of differentiation. Differentiation of the temporal average of rotation rate, shows that the radial gradient is mainly concentrated in the outer shear layer and in the tachocline, while the latitudinal gradient is of course, confined to the convection zone (Antia et al. 2008). It is difficult to determine the radial gradient in the tachocline region reliably, as the thin tachocline region is not adequately resolved in inversion results. Once again there are significant differences in these gradients between the GONG and MDI results, which are largely cancelled while calculating the temporally varying components. Fig. 5 shows the radial $\left(\Omega_{r}=\partial \Omega / \partial r\right)$ and latitudinal gradients $\left(\Omega_{\theta}=(1 / r) \partial \Omega / \partial|\theta|\right)$ at $r=0.95 R_{\odot}$ at a few selected latitudes. It can be easily seen that temporal variations in these gradients are a sizable fraction of their average values, up to or exceeding $20 \%$. This is much larger than the relative variation of the order of $0.5 \%$ in the rotation rate. This substantial variation in the shear should play some role in the solar dynamo.

Temporal variations in these gradients also show bands of higher and lower than average gradients similar to those of zonal flows. These bands are also correlated to the location of sunspots in the butterfly diagram (Antia et al. 2008). It is found that the sunspots are predominantly formed in low-latitude regions where the variation of the radial gradient is positive and that of the latitudinal gradient is negative. Since both these gradients are negative, it means that sunspots tend to occur in regions of reduced radial shear but enhanced latitudinal shear.

\section{References}

Ahmad, Q. R., et al. 2002, PRL, 89, 011301

Anders, E. \& Grevesse, N. 1989, Geochimica et Cosmochimica Acta, 53, 197

Antia, H. M. 1998, AESA, 330, 336

Antia, H. M. \& Basu, S., 2000, ApJ, 541, 442

Antia, H. M. \& Basu, S., 2001, ApJ, 559, L67

Antia, H. M. \& Basu, S. 2005, ApJ, 620, L129

Antia, H. M. \& Basu, S. 2006, ApJ, 644, 1292

Antia, H. M., Basu, S., \& Chitre, S. M., 2008, ApJ, 681, 680

Asplund, M., Grevesse, N., Sauval, A. J., Allende Prieto, C., \& Kiselman, D. 2004, A\&A, 417, 751 (Erratum in $2005 A \mathscr{E} A, 435,339$ )

Asplund, M., Grevesse, N., \& Sauval, A. J. 2005, in Cosmic Abundances as Records of Stellar Evolution and Nucleosynthesis, eds., T. G. Barnes, F. N. Bash, ASP Conf. Ser. 336, p. 25 (AGS05)

Ayres, T. R. 2008, ApJ, 686, 731

Badnell, N. R., Bautista, M. A., Butler, K., Delahaye, F., Mendoza, C., Palmeri, P., Zeippen, C. J., \& Seaton, M. J. 2005, MNRAS, 360, 458

Bahcall, J. N. \& Pinsonneault, M. H. 2004, PRL, 92, 121301

Bahcall, J. N., Serenelli, A. M., \& Pinsonneault, M. 2004, ApJ, 614, 464

Bahcall, J. N., Basu, S., Pinsonneault, M., \& Serenelli, A. M. 2005a, ApJ, 618, 1049

Bahcall, J. N., Basu, S., \& Serenelli, A. M., 2005b, ApJ, 631, 1281

Bahcall, J. N., Serenelli, A. M., \& Basu, S. 2006, ApJS, 165, 400

Basu, S. \& Antia, H. M. 1995, MNRAS, 276, 1402

Basu, S. \& Antia, H. M. 1997, MNRAS, 287, 189

Basu, S. \& Antia, H. M., 2003, ApJ, 585, 553 
Basu, S. \& Antia, H. M., 2004, ApJ, 606, L85

Basu, S. \& Antia, H. M., 2008, Phys. Rep., 457, 217

Brown, T. M. \& Christensen-Dalsgaard, J. 1998, ApJ, 500, L195

Brun, A. S., Turck-Chièze, S., \& Zahn, J.-P. 1999, ApJ, 525, 1032

Caffau, E., Ludwig, H.-G., Steffen, M., Ayres, T. R., Bonifacio, P., Cayrel, R., Freytag, B., \& Plez, B., 2008, A\&A, 488, 1031

Castro, M., Vauclair, S., \& Richard, O. 2007, A\&A, 463, 755

Centeno, R. \& Socas-Navarro, H. 2008, ApJ, 682, L61

Chaplin, W. J., Serenelli, A. M., Basu, S., Elsworth, Y., New, R., \& Verner, G. A. 2007, ApJ, 670,872

Christensen-Dalsgaard, J. 2002, Rev. Mod. Phys., 74, 1073

Christensen-Dalsgaard, J. \& Gough, D. O. 1980, Nature 288, 544

Christensen-Dalsgaard, J., Proffitt, C. R., \& Thompson, M. J. 1993, ApJ, 403, L75

Christensen-Dalsgaard, J., et al., 1996, Science 272, 1286

Covas, E., Tavakol, R., Moss, D., \& Tworkowski, A., 2000, A\&\&A, 360, L21

Delahaye, F. \& Pinsonneault, M. H. 2006, ApJ, 649, 529

Drake, J. J. \& Testa, P. 2005, Nature 436, 525

Feldman, U. \& Widing, K. G. 2003, Space Sci. Rev., 107, 665

Garcia-Alvarez, D., Drake, J. J., \& Testa, P. 2008, ArXiv:0808.1794

Gough, D. O. 1984, Mem. Soc. Astron. Ital., 55, 13

Gough, D. O., et al., 1996, Science, 272, 1296

Grevesse, N. \& Noels, A. 1993, in Origin and Evolution of the Elements, eds., N. Prantzos, E. Vangioni-Flam, M. Cassè, Cambridge Univ. Press, p. 14

Grevesse, N. \& Sauval, A. J. 1998, Space Sci. Rev., 85, 161 (GS98)

Guzik, J. A., Watson, L. S., \& Cox, A. N. 2005, ApJ, 627, 1049

Hill, F., et al., 1996, Science 272, 1292

Howard, R. F. \& LaBonte, B. J., 1980, ApJ, 239, L33

Howe, R., Christensen-Dalsgaard, J., Hill, F., Komm, R. W., Larsen, R. M., Schou, J., Thompson, M. J., \& Toomre, J., 2000, ApJ, 533, L163

Howe, R., Rempel, M., Christensen-Dalsgaard, J., Hill, F., Komm, R. W., Larsen, R. M., Schou, J., \& Thompson, M. J., 2006, Astrophys. J. 649, 1155

Iglesias, C. A. \& Rogers, F. J. 1996, ApJ, 464, 943

Rogers, F. J. \& Iglesias, C. A. 1992, ApJS, 79, 507

Scherrer, P. H., et al., 1995, Solar Phys., 162, 129

Schmelz, J. T., Nasraoui, K., Roames, J. K., Lippner, L. A., \& Garst, J. W., 2005, ApJ, 634, L197

Schou, J., Kosovichev, A. G., Goode, P. R., \& Dziembowski, W. A., 1997, ApJ, 489, L197

Schou, J., et al., 1998, ApJ, 505, 390

Schou, J., et al., 2002, ApJ, 567, 1234

Sivaraman, K. R., Antia, H. M., Chitre, S. M., \& Makarova, V. V. 2008, Solar Phys., 251, 149

Snodgrass, H. B., 1987, Solar Phys., 110, 35

Spiegel, E. A. \& Zahn, J.-P., 1992, A\&A, 265, 106

Thompson, M. J., et al., 1996, Science, 272, 1300

Turck-Chièze, S., Couvidat, S., Piau, L., Ferguson, J., Lambert, P., Ballot, J., García, R. A., \& Nghiem, P., 2004, PRL, 93, 211102

Ulrich, R.K., Boyden, J.E., Webster, L., Snodgrass, H.B., Padilla, S.P., Gilman, P., \& Shieber, T., 1988, Solar Phys., 117, 291

Vorontsov, S. V., Christensen-Dalsgaard, J., Schou, J., Strakhov, V. N., \& Thompson, M. J. 2002, Science, 296, 101

Young, P. R. 2005, A\&A, 444, L45

\section{Discussion}

BOCHSLER: I am surprised to see how astrophysicists can spend their time using abundances of high-FIP elements $(\mathrm{Ne} / \mathrm{O} / \mathrm{Ar})$ as free parameters, despite the fact that these 
abundances have been reliably determined by many independent methods and their systematics is well understood (e.g. Young 2005, Bochsler 2007 (A\&A), Lodden 2008, ApJ.).

ANTIA: Unfortunately, different techniques of measuring elemental abundances give different results and that is why the current controversy has arisen. Hence, we need to check the effect of the range of abundances on other models. If all measurements of solar abundances give the same result this may not be required. 